Digital Press Social Sciences and Humanities

Le rôle des réseaux culturels officiels au sein de la diplomatie culturelle française à Banyumas

Tri Asiati

Proceeding of Conférence internationale sur le français 2018

Joesana Tjahjani, Merry Andriani, Sajarwa, Wening Udasmoro (eds) 


\title{
Le rôle des réseaux culturels officiels au sein de la diplomatie culturelle française à Banyumas
}

\author{
Tri Asiati \\ Universitas Jenderal Soedirman, Jawa Tengah, Indonesia \\ e-mail : asiatitri@yahoo.com
}

\section{Résumé}

Depuis le XIIème siècle, la culture française est importante aux yeux du monde de par son excellence, notamment avec son influence sur la langue, la littérature, la philosophie et les courants de pensée. Elle représente tout un art de vivre, marqué par des valeurs traditionnelles, parmi lesquelles une gastronomie appréciée, et une constante reconnaissance de sa haute couture. Elle joue un rôle uniquement dans la diplomatique française qui comporte de l'action politique, économique, et culturelle. Cette action historiquement est commencée par le régime colonial hollandais et elle est fondamentalement renforcée aujourd'hui à travers les réseaux culturels officiels. Dans ce cas, la problématique se focalise de leurs fonctions et de leurs actions face à la communauté Banyumas en tant qu'une nouvelle relation qui est confrontée à des conditions géographiques, sociales, et culturelles. En se réfère à la diplomatie culturelle française à cette région, cette recherche met l'accent sur la méthode descriptive qualitative à l'égard de décrire et d'analyser les données avec l'objectif d'expliquer de l'action diplomatique à travers de la culture française.

\section{Mots-clés}

culture, diplomatie, française, réseaux culturels, et rôle

\begin{abstract}
Since the 12th century, French culture has been important to the world because of its excellence, particularly with its influence on language, literature, philosophy and streams of thought. It represents an art of living, marked by traditional values, among which is the highly appreciated gastronomy, and a constant recognition of its haute couture. It plays a role only in French diplomacy that involves political, economic, and cultural action. This action is historically started by the Dutch colonial regime and is fundamentally reinforced today through official cultural networks. In this case, the problematic focus of their functions and their actions in the face of the Banyumas community as a new relationship that is confronted with geographical, social, and cultural conditions. Referring to French cultural diplomacy in this region, this research focuses on the qualitative descriptive method of describing and analyzing data with the objective of explaining diplomatic action through the French culture.
\end{abstract}

\section{Keywords}

Culture, diplomacy, French, cultural networks, role

\section{Introduction}

D’après l'Organisation des nations unies pour l'éducation, la science et la culture (UNESCO, 2007), la culture dans son sens large est considérée comme l'ensemble des traits distinctifs, spirituels et matériels, intellectuels et affectifs, qui caractérisent une société et un groupe social. Elle englobe, outre les arts et les lettres, les modes de vie, les droits fondamentaux de l'être humain, les systèmes de valeurs, les traditions et les croyances. Tandis que Renaud Donnedieu de Vabres dans Loïc (2008, p. 1) donne de son avis que la 
culture est un antidote à la violence, car elle nous invite à la compréhension d'autrui et féconde la tolérance, en nous incitant à partir à la rencontre d'autres imaginaires et d'autres cultures. Il souligne aussi sur la dimension contemporaine de la culture qui passe par l'échange entre les différents découpages du globe et le fait qu'elle est devenue un mode moderne de confrontation entre états, un processus nouveau d'exaltation et d'expression du sentiment national. La culture et donc l'art en général touchent un vaste champ de domaines et de ce fait, il est incontestable qu'on peut leur attribuer un lien avec le pouvoir.

Si la culture est un domaine qui depuis longtemps est lien au pouvoir, elle se relie à la politique et à l'économie. Cela se réfère uniquement au ancien Europe selon Loïc (2008, pp. 9-10) que la dimension de subordination de la culture à la religiosité comme à l'époque des arts primitifs que l'art est une prise de conscience de l'homme de sa propre existence et de son pouvoir d'agir sur le monde. Cependant, la fin de la suprématie grecque que le monde latin domine l'Occident, la valeur d'un objet artistique comme le butin à l'ennemi devient un intérêt très important avec la domination du monde romain. Ensuite, au moment d'Auguste, la culture se présente comme le souverain hellénistique qu'il veut reprendre l'image l'Alexandre Le Grand. En fin, la diffusion progressive du christianisme considère que l'œuvre reste alors une transcription d'une volonté religieuse par le biais d'un homme et elle est continuée au XIIIème siècle par les artistes qui utilise la signature comme le signe aristocratique et le symbole sacré. Nous nous apercevons donc que la culture peut être envisagée dans un contexte pouvoir en tant qu'un instrument diplomatique.

Quand nous envisageons la culture à l'instar de l'instrument diplomatique (soft power) ou pouvoir d'attraction dans le monde international, la France est une grande puissance en Europe qui la présente à partir de la guerre Franco-prussienne en 1870. Cette stratégie est simplement prise de reconstruire son image en tant qu'une nation civilisée pour répondre à sa défaite dans cette guerre. Ce pour cela, la naissance de l'Alliance Française est une action culturelle extérieure française que son objectif favorise de la langue française comme une langue civilisatrice et ce réseau est noté aujourd'hui comme la première institution culturelle au monde.

La présente d'Alliance Française au monde est un point de départ de la France pour lancer des réseaux institutionnels qui sont intégrés au sous du ministère des Affaires étrangères qu'ils représentent de l'hexagone en extérieure. Ils sont considérés comme un état objet d'organisations centralisatrices telles que l'ex Agence Française d'action Artistique (AFAA) devenue l'agence cultures France qui est en fait placée sous l'égide principale du ministère des Affaires étrangères (MAE) . Ce sont les Centres Culturels Français, l'Institut Français, le Campus France, et les lycées Français à l'étranger que leurs missions font parties de promotion et d'enseignement de la culture française sur toute la surface du globe.

En soulignant que la mission des réseaux culturels officiels français sur toute la surface du globe comporte de l'aspect politique international que le résultat nous montre aujourd'hui en tant qu'une grande influence du monde. Cette politique culturelle se réalise à la base de la fondation de l'Organisation International de la Francophonie (OIF) qui réunifie à tous les états au sous de la langue française. Cette organisation supporte du pouvoir de la langue française à l'égard d'une langue uniquement du monde qui respecte de l'hétérogénéité culturelle. En conséquence, la présence des réseaux culturels officiels français implante absolument aux pays francophones ou bien aux pays francophiles.

Nous citons que l'existence des réseaux culturels officiels français a déjà implanté aux pays francophones ou bien aux pays francophiles. Chaque réseau fait face des différences problématiques qui se relient souvent à l'espace de la France dans la position géopolitique et à la situation de l'état. Nous prouvons cette situation en Indonésie en tant qu'un pays non-francophone, mais elle a particulièrement dans le développement de la culture française grâce à la coopération bilatérale Franco-Indonésienne. Nous trouvons qu'il y en a plusieurs réseaux culturels officiels s'installent aux capitales de la ville comme à Jakarta, Medan, Bandung, Yogyakarta, Surabaya, Bali et Balikpapan. De plus, l'Institut Français Indonésie en 2012 a déjà lancé du programme Warung Prancis à l'égard de la coopération universitaire que son rôle est renforcé la coopération éducative, culturelle, et linguistique qui peuvent plus toucher la société académique. Par conséquence, la culture française est considérée comme la partie de la société indonésienne aujourd'hui.

Dès que l'Institut Français Indonesia (IFI) a déjà lancé le programme de Warung Prancis (2018), la diplomatie culturelle française est bien valorisée, notamment à la région éloignée. Il ressemble aux réseaux culturels officiels qui s'accordent de l'objective de la coopération entre l'IFI et l'université. Sa fonction est liée à l'objectif de l'université et à plus autonome. Dans ce cas-là, nous voudrions analyser le rôle de Warung Prancis, notamment qui se trouve à l'université Jenderal Soedirman (UNSOED) en tant que l'un des établissements culturels français. Il prend particulièrement son rôle à cause de la position uniquement à la région Banyumas qui se trouve au loin de la capitale. Finalement, nous allons l'analyser en tant qu'un agent culturel français à Banyumas par la méthode descriptive qualitative à l'égard de 
décrire et d'analyser les données avec l'objectif d'expliquer de l'action diplomatique à travers de la culture française à cette région

\subsection{Diplomatie culturelle française}

La diplomatie culturelle est un thème canonique qui met en lumière l'importance de la culture dans la compréhension et l'explication des phénomènes politiques et stratégiques internationaux. Robert Frank (2012) affirme que la diplomatie culturelle comporte trois éléments principaux, tels qu'ils soient la recherche de la paix, le besoin d'affirmer une identité, la puissance ou l'influence. Cette idée se focalise de ne pas faire la guerre, de promouvoir la paix par la diplomatie culturelle de tous les pays du monde ne servent initialement pas à déclencher la guerre.

En particulier, à partir du XXe siècle dans le contexte de la mondialisation, les échanges culturels occupent une place de plus en plus importante dans les relations internationales. La culture n'agit pas seulement comme variable causale, mais également comme instrument d'une stratégie politique. Cependant qu'Ory $(2011$, p. 114) affirme que l'action culturelle demeure un instrument privilégié pour façonner un environnement favorable aux prises de décision d'un État. Les États utilisent les moyens culturels pour valoriser leur culture, pour promouvoir leurs valeurs et rehausser leur influence dans la communauté internationale. D'une part, les gouvernements considèrent qu'il est sa responsabilité de défendre la culture nationale à l'intérieur, de protéger leur territoire et leur population des flux étrangers et de promouvoir cette culture nationale à l'extérieur par tous les canaux possibles. D'autre part, ils se trouvent dans une concurrence commerciale, où la dissuasion de l'arme atomique recule le risque d'une guerre mondiale. Pour renforcer leur puissance, les États cherchent évidemment à utiliser toute forme d'action, y compris l'action culturelle. Dans cette perspective, la diplomatie culturelle, en tant que stratégie étatique, composante de la politique extérieure est affichée sur l'agenda politique de nombreux gouvernements. Delaunay (2007, p. 43) ajoute aussi sur la quatrième dimension de la politique extérieure après les années 1960 telles que la politique, le militaire, l'économique et la diplomatie culturelle qui est entrée de plein fouet dans les dispositifs de représentation extérieure des nations comme la France, les Etats-Unis, et la Grande-Bretagne.

En ce qui concerne de la diplomatie culturelle qui est née en Europe, elle s'est développée parallèlement aux confrontations politiques et militaires entre les grandes puissances comme la France et la Grande-Bretagne. La France déploie d'une part sa stratégie diplomatie culturelle comme une tradition de propager ses valeurs messianiques et ses valeurs universelles. Mais d'autre part, la culture sert d'autre terrain pour qu'elle puisse compenser ses faiblesses sur le plan économique dans le combat de l'influence. Chaubet $(2014 / 2018)$ ajoute qu'il y a trois grandes étapes dans le fonctionnement de l'action culturelle extérieure française historiquement par la mise en place du réseau d'institutions françaises à l'étranger en 1883, et puis la fondation des comités des Alliances françaises en tant qu'un premier institut français à la veille au sein de la Première Guerre mondiale à Florence en 1906, et l'existence des lycées français à l'entre les deux-guerres.

Nous soulignons que les grandes étapes de l'action culturelle extérieure française sont contribuées au pouvoir extérieurement de la France jusqu'à ce qu'elles sont institutionnalisées au sous des Ministères des Affaires Étrangères. Dans ce cas-là, l'état renforce des agents culturels ce que nous connaissons aujourd'hui tels que l'Alliance Française et l'Institut Français. Les deux établissements font partis fondamentalement du promouvoir la culture et la langue française en extérieure que l'un et l'autre sont des caractéristiques différences, notamment de la structure d'organisation. L'Alliance Française montre la situation hétérogène à cause de son statut qui est plus associatif des alliances qu'elle fonctionne sur la base des réseaux locaux d'animateurs et est subventionnée par les Ambassades en fonction de leurs activités. Dans le meilleur des cas, des personnalités francophones enthousiastes arrivent à faire rayonner les activités, en étoffant une offre de cours de qualité, en menant des activités de diffusion culturelle et en diffusant, via le livre et les médiathèques, notre culture. Alors que l'Institut Français est juridiquement partie des services extérieurs des Ambassades de France qu'il possédé de l'autonomie financière. Ils ont trois missions systématiquement telles que : Culture (diffusion et aide à la création), Cours (enseignement du français général et spécialisé), médiathèque (Diffusion de notre culture via le livre et les nouveaux supports tels DVD, site internet, etc.). De plus, cette idée est celle d'une synergie entre les trois composantes très liées. En conclure qu'ils sont des outils différents et leurs missions sont proches mais avec des règles de fonctionnement pour la mise en œuvre de ces missions assez dissemblables. D'un côté celle de la puissance publique qui cadre le fonctionnement et de l'autre est une souplesse due au système associatif. À la différence des autres réseaux culturels étrangers (Cervantès, Goethe Institut, British Council), la France ne possède pas de "label unique" et connaît donc un double système qui voit la coexistence des Alliances et des Instituts. 
En Indonésie, l'Ambassade de France met l'accent sur sa stratégie culturelle diplomatie par le programme de Warung Prancis qu'elle a déjà lancé en 2012 à l'égard du promouvoir de l'éducation, de la culture et de la langue française. Ce programme est implanté à l'université partenaire à travers leur coopération académique que l'Institut Français Indonesia a noté de 31 universités partenaires. Finalement, ce réseau culturel supporte de la France pour valoriser son influence à travers l'ambiance académique.

\section{Méthode}

La méthode désigne la manière d'agir en suivant un ordre logique. Elle signifie aussi l'ensemble de procédés permettant de parvenir à un résultat. D’après le Dictionnaire Junior Larousse (2013:637) la méthode est un manuel où sont présentées de manière progressive les règles à suivre pour apprendre une technique, une science ou un art. Dans cette recherche nous utilisons la méthode descriptive qualitative que Margot Phaneuf (2013) a déjà exprimé sur la méthode descriptive-qualitative est un schéma de recherche qui visent à faire une interrogation globale sur des phénomènes humains complexes afin de comprendre les interactions et l'influences internes et externes qui les modifient dans leur milieu naturel. Tandis que nous expliquons aussi sur l'analyse des données qui se focalise de: (1) classification des données, (2) vérification auprès, (3) identification de profils de comportements, (4) élaboration d'une théorie en fonction du groupe étudié et peut-être d'une certaine manière généralisable, si l'échantillon est important.

\section{Résultats et discussions}

Banyumas est une région particulière au Java Central grâce à la tradition unique qu'elle se rencontre à la culture Javanaise et à celle Sundanaise. Cette région se trouve au loin de la capitale et elle devient le centre des autres villes. De plus, sa richesse naturelle supporte du développement régional. Dans ce caslà, cette condition particulière renforce de la naissance de l'université publique ce que nous appelons Universitas Jenderal Soedirman (UNSOED).

Dès que l'existence de l'université Jenderal Soedirman à la région Banyumas, elle met l'accent sur le développement académique à cette région. Sa contribution n'est qu'absolument dans le point académique, mais elle est contribuée aussi dans la coopération internationale, notamment dans la valorisation linguistique. Elle se sert la société Banyumas à la transformation culturelle qui est provoquée par la mondialisation. L'une des coopérations internationales est une coopération académique entre cette université et l'Institut Français Indonesia (IFI) pour fonder Warung Prancis en 2014. Cette coopération se focalise du promouvoir de la culture et la langue française à la communauté Banyumas. Par conséquence, Elle devient le réseau culturel officiel qui connecte la société Banyumas à la culture et à la langue française premièrement.

Nous envisageons que Warung Prancis est l'une des services d'Institut Français Indonésie qui s'oriente vers du promouvoir de la culture et la langue français. Elle détermine le rôle des réseaux culturels officiels à la région éloignée que l'IFI ne peut pas s'occuper pleinement de la société. À vrais dire qu'il est comme une institution autonome plus particulière, parce qu'il est une place de l'échange culturel entre la culture locale et la culture française. De plus, cet établissement s'approche facilement de la société à travers de l'ambiance académique. Nous montrons qu'il peut jouer un rôle dans sa mission, surtout à la région Banyumas à partir de la recherche descriptive qualitative.

À partir de la recherche qui se focalise du rôle de Warung Prancis au sein de la diplomatie culturelle française à la région Banyumas, nous partageons les étapes de la recherche. En avant, nous demandons à 50 répondants pour répondre 50 questions à travers le réseau culturel français à cette région. Et puis, nous citons les questions qui montrent des aspects réels sur la fonction des établissements culturels officiels français. Et en fin, nous tirons que l'existence de Warung Prancis à cette région est contribuée au promouvoir de la culture et la langue français en se basant sur le résultat de la réponse.

Tableu 1 Connaissance de bureau culturelle en Indonésie

\begin{tabular}{|l|l|l|l|l|l|l|l|l|l|l|l|l|l|}
\hline No & Questions & 1 & 2 & 3 & $\ldots$ & 19 & 20 & 21 & 22 & 23 & 24 & 25 & A \\
\hline 40 & $\begin{array}{l}\text { Instituts } \\
\text { Culturels } \\
\text { Français en }\end{array}$ & & & & & & & & & & & & \\
\end{tabular}




\begin{tabular}{|c|c|c|c|c|c|c|c|c|c|c|c|c|}
\hline Indonésie & & & & & & & & & & & & \\
\hline $\begin{array}{ll}\text { a. } & \text { École } \\
& \text { Français } \\
& \text { d'Extrême- } \\
& \text { Orient } \\
\end{array}$ & & & & & & & & & & & & \\
\hline $\begin{array}{ll}\text { b. } & \text { Institut } \\
& \text { Français } \\
& \text { Indonesia } \\
\end{array}$ & $\mathbf{X}$ & $\mathbf{X}$ & $\mathbf{X}$ & $\mathbf{X}$ & $\mathbf{X}$ & $\mathbf{X}$ & $\mathbf{X}$ & $\mathbf{X}$ & $\mathbf{X}$ & $\mathbf{X}$ & & 23 \\
\hline $\begin{array}{ll}\text { c. } & \text { Campus } \\
& \text { France } \\
\end{array}$ & & & & & & & & & & & $\mathbf{X}$ & 2 \\
\hline
\end{tabular}

Tableu 2 Connaissance de bureau culturelle dans le quartier des correspondants

\begin{tabular}{|c|c|c|c|c|c|c|c|c|c|c|c|c|c|}
\hline \multirow{5}{*}{$\begin{array}{l}\text { No } \\
41\end{array}$} & Questions & 1 & 2 & 3 & $\ldots$ & 19 & 20 & 21 & 22 & 23 & 24 & 25 & $\mathrm{~A}$ \\
\hline & $\begin{array}{l}\text { Ya-t-il des } \\
\text { instituts de } \\
\text { coopération } \\
\text { académique et } \\
\text { culturelle } \\
\text { françaises dans } \\
\text { votre région } \\
\end{array}$ & & & & & & & & & & & & \\
\hline & a. Oui & $\mathbf{X}$ & $\mathrm{X}$ & & & $\mathbf{X}$ & $\mathrm{X}$ & $\mathbf{X}$ & $\mathbf{X}$ & $\mathbf{X}$ & $\mathbf{X}$ & $\mathbf{X}$ & 23 \\
\hline & b. Non & & & & & & & & & & & & 1 \\
\hline & c. Ne sais pas & & & & & & & & & & & & 2 \\
\hline
\end{tabular}

Tableu 3 Quantité d'activité pédagogique et culturelle français à Banyumas

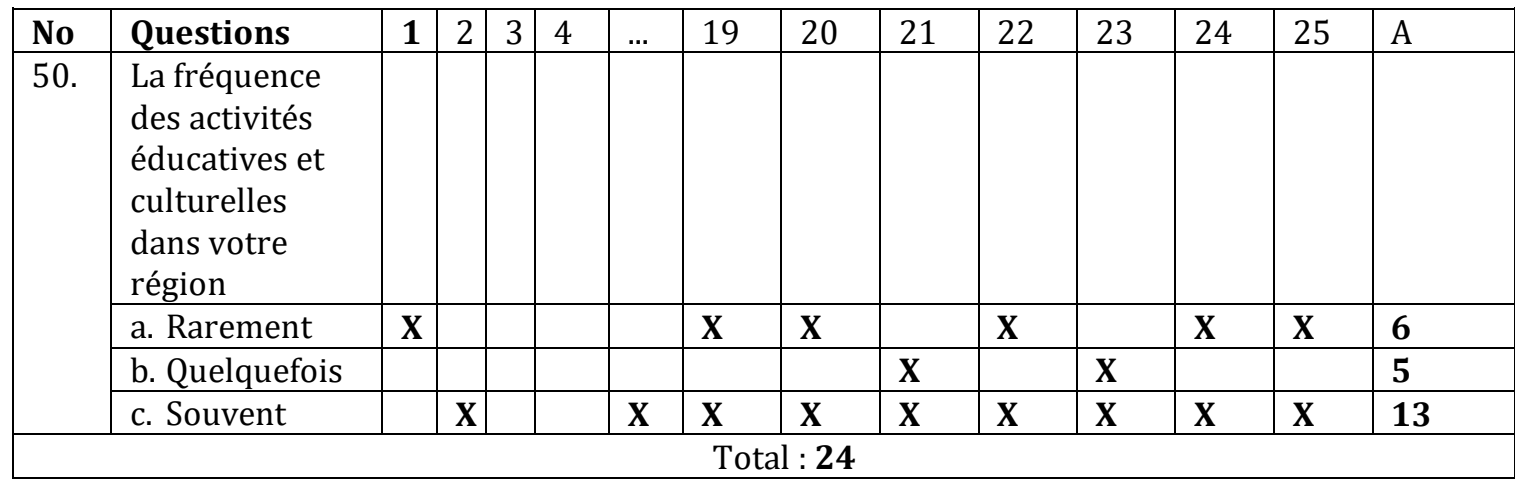

Ces tableaux prouvent que Warung Prancis est contribué au développement de la culture et de la langue française à cette région. Dans ce cas-là, les répondants expriment leurs connaissances sur cet établissement à partir de la connaissance des réseaux culturels officiels. Les répondants expriment que l'Institut Français Indonésie est un établissement culturel officiel français, si bien qu'ils connaissent des autres établissements tels que : le Campus France et l'École Français d'Extrême-Orient. Ensuite, Warung Prancis est un premier établissement français qui pilote de la valorisation de la culture et de l'enseignement du français qu'il est considéré comme une association francophonie à cette région. Par conséquence, le résultat du sondage exprime que Warung Prancis est un réseau culturel français à Banyumas qui contribué au promouvoir de la culture et de la langue française.

Grâce à l'existence de Warung Prancis, la société Banyumas connait de la culture et de la langue française, parce qu'il organise les activités culturelles et les activités académiques. Toutes les activités sont supportées par l'Institut Français Indonésie en tant qu'un réseau partenaire qu'ils ont la même mission au sein de présenter la France globalement. Cet établissement prend un rôle de la transformation sociale de la culture locale à la culture internationale à cette région. Par conséquence, la diplomatie culturelle française est bien implantée en Indonésie à cause du programme de Warung Prancis à l'instar du petit réseau culturel officiel qui est plus spécifique, parce qu'il est comme une place de l'échange culturel 
entre la culture française et la culture locale.
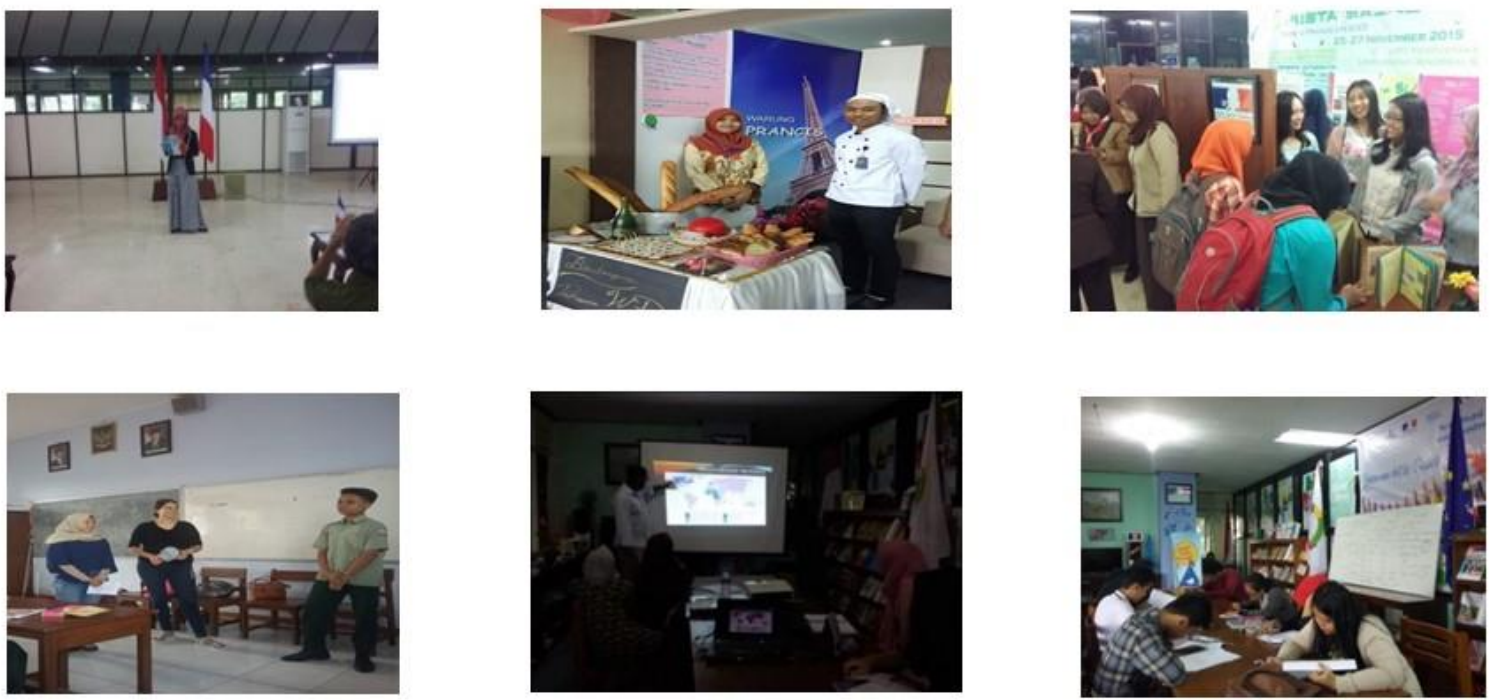

Figure 1 Des activités de Warung Prancis UNSOED

\section{Conclusion}

La diplomatie culturelle est un thème canonique qui met en lumière l'importance de la culture dans la compréhension et l'explication des phénomènes politiques et stratégiques internationaux. Cette idée se focalise des échanges culturels qui occupent une place de plus en plus importante dans la relation internationale. Dans cette perspective, la diplomatie culturelle, en tant que stratégie étatique, composante de la politique extérieure est affichée sur l'agenda politique de nombreux gouvernements. L'une des grandes puissances du monde, la France met l'accent sur trois grandes étapes dans le fonctionnement de l'action culturelle extérieure française par la mise en place du réseau d'institutions françaises à l'étranger, la fondation des comités des Alliances françaises, et l'existence des lycées français. Dans ce cas-là, l'état renforce des agents culturels ce que nous connaissons aujourd'hui tels que l'Alliance Française et l'Institut Français. Les deux établissements font partis fondamentalement du promouvoir la culture et la langue française en extérieure que l'un et l'autre sont des caractéristiques différences, notamment de la structure d'organisation.

En ce qui concerne de l'implantation culturelle officielle de la France, l'Ambassade de France en Indonésie renforce bien du promouvoir de sa culture et de sa langue par le programme de Warung Prancis qu'elle a déjà lancé en 2012. Sa fondation supporte de l'Institut Français et le Campus France pour valoriser de la particularité de la France au sein des domaines académique, culturelle, et linguistique à travers l'université partenaire qui peut plus toucher les académiciens ou la société locale. Cette idée s'implante bien à l'université Jenderal Soedirman où elle se trouve à la région Banyumas. Cette association est considérée comme un réseau culturel officiel français premièrement qu'il se sert à la connaissance culturelle et linguistique au public. De plus, il ressemble à la clé de l'échange culturel pour la société Banyumas qui est en train d'entrer dans la mondialisation. Finalement, son existence supporte fondamentalement de la diplomatie culturelle qui prend de la grande vision sur la paix et la diversité culturelle à tous les pays du monde.

\section{Références}

Chaubet, F. (2004). L'Alliance française ou la diplomatie de la langue (1883-1914). Revue historique,

$$
\text { 632(4), 763-785. https://doi.org/10.3917/rhis.044.0763 }
$$

Chaubet, F. (2018). L'action culturelle française dans le monde : 150 ans d'expériences. Retrieved from https://chmcc.hypotheses.org/2725 
Institut Français Indonesia. (2018). Warung Prancis. Retrieved from https://www.ifi-id.com/id/warungprancis

Loïc, G. (2008). La diplomatie culturelle française : La culture face à de nouveaux enjeux ? IEP de Toulouse, Toulouse.

Ory, P. (2011). Les relations culturelles internationales au XXe siècle, de la diplomatie culturelle à l'acculturation. Brussels: Peter Lang.

Phaneuf, M. (2013). La recherche en soin infirmières en 3ème Module. Retrieved from www.prendre soin.org/wp-content/upload/2013/09/3_MéMéthodes-qualitatives.pdf

Robert, F. (2012). Pour l'histoire des relations internationales. Paris: PUF.

Roche, F., \& Delaunay, J.-M. (2007). Diplomaties culturelles et décadences nationales : réflexions sur le XXe siècle. In F. Roche (Ed.), Géopolitique de la culture, Espace d'identité, Projections, Coopérations (p. 43). Paris: L'Harmattan.

UNESCO. (2017). Apprendre À Vivre Ensemble. Retrieved from http://www.unesco.org/new/fr/socialand-human-sciences/themes/international-migration/glossary/cultural-diversity/ 